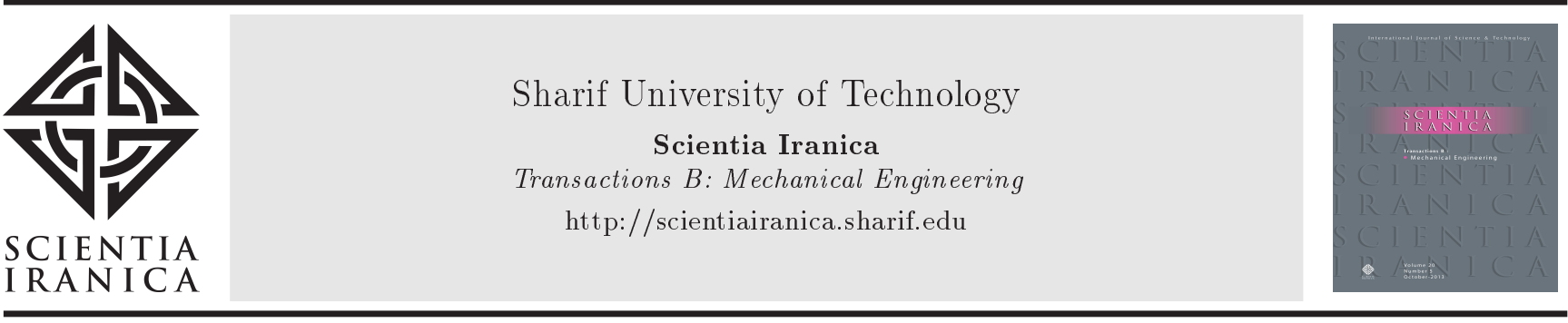

Research Note

\title{
A numerical investigation of synthetic jet effect on dynamic stall control of oscillating airfoil
}

\author{
A. Shokrgozar Abbasi* and Sh. Yazdani \\ Department of Mechanical Engineering, Payame Noor University, Iran.
}

Received 24 February 2019; received in revised form 19 June 2019; accepted 12 October 2019

\author{
KEYWORDS \\ Dynamic stall; \\ Oscillating airfoil; \\ Flow control; \\ Synthetic jet actuator; \\ Numerical solution; \\ Momentum \\ coefficient.
}

\begin{abstract}
At high angles of attack, the dynamic stall phenomenon can arise from the vortex shedding, particularly in an oscillating airfoil. As a result of this phenomenon, a considerable decrease in the lift and an increase in the drag and pitching moment coefficients are observed. This study aims to investigate the flow control of an NACA 0015 airfoil using a Synthetic Jet (SJ). The flow was assumed to be unsteady and turbulent at the Mach number of 0.2 and Reynolds number of 1 million. This research was conducted at the angle of attack of $15^{\circ} \pm 10^{\circ}$. In order to carry out the numerical analysis of the problem, the 2D compressible turbulent Navier-Stokes equations based on "Roe" scheme with the second-order accuracy were solved. Turbulence modeling was carried out using the threeequation $k-k_{L}-\omega$ model. According to the obtained results, this flow control method could significantly control or eliminate the dynamic stall of the airfoil. In addition, the phase difference between the jet and airfoil oscillations was mostly affected by the dynamic stall decrement. In these changes, using SJ with a momentum coefficient of 0.1 brought about the amplitude of maximum lift at $\varphi=-30^{\circ}$, and the multiplication of the coefficients of drag and moment amplitudes at $\varphi=-10^{\circ}$ ensured the best performance.
\end{abstract}

(C) 2021 Sharif University of Technology. All rights reserved.

\section{Introduction}

Dynamic stall has many severe consequences and it should be anticipated as quickly as possible. At high angles of attack, flow separation may occur. In addition, when flow is not controlled, dynamic stall occurs at a specific angle. As a result of uncontrolled flow, the lift and drag forces would suddenly decrease and increase, respectively, and the wing loses stability.

\footnotetext{
*. Corresponding author. Tel.: +985138683887; Fax: +98 5138451620;

E-mail address: shokrgozar.ali@gmail.com (A. Shokrgozar Abbasi)
}

doi: $10.24200 /$ sci. 2019.52743 .2870
Thus, flow control is of significance when it comes to preventing such difficulties. In recent years, many experimental and numerical investigations have been conducted to propose an acceptable method that can inhibit the separation flow and control the stall [1-4]. Duvigneau and Visonneau [5] numerically studied the effects of Synthetic Jet (SJ) control on the NACA 0015 airfoil at $\operatorname{Re}=8.96 \times 10^{5}$. They considered the jet frequency of 0.748 , non-dimensional jet velocity of 1.72 , and the inclined angle of $25^{\circ}$ in their study. The effect of a tangential SJ on aerodynamic characteristics of a NACA 23012 airfoil was also investigated by Esmaeili et al. [6]. They concluded that at the chord Reynolds number of $\operatorname{Re}=2.19 \times 10^{6}$, two jet oscillating frequencies with different blowing ratios could be obtained and it can be stated that the activation of the SJ could 
control the stall characteristics of the airfoil. Zhang et al. [7] also investigated the effect of suction control on NACA 0012 through LES methods and concluded that by increasing the suction coefficient, the lift-drag ratio would first increase and then, decrease. In addition, they estimated the location of suction and found that the only area with a considerable control effect on flow separation and lift increase was behind the separation point. Tran et al. [8] investigated the ability of dynamic large eddy simulation to predict the flow interactions of a finite-span SJ on NACA 4421 airfoil. Moreover, they compared the results of the large eddy simulation with those of previous experiments and direct numerical simulations. Montazer et al. [9] conducted a numerical study that investigated the effect of the SJ on NACA 0015 at Reynolds number of $\operatorname{Re}=896000$. They aimed to optimize the jet implementation to improve the aerodynamic characteristics of the airfoil. The results of their study indicated that the jet implementation was the most useful technique for post-stall angle and could increase the Lift-to-Drag $(L / D)$ by $66 \%$. Moreover, Tran et al. [10] numerically investigated the effect of the SJ on S809 airfoil by considering the low-energy input requirements. According to their study, jet control, installed near the leading edge, could reduce the flow separation and, consequently, reduce the hysteresis by $73 \%$. Yousefi et al. [11] studied the effects of blowing and suction flow control on NACA 0012. In fact, they explored the effects of the width jet. It was observed that the lift-to-drag ratio could be improved with an increase in the suction and blowing jet width. Furthermore, Moshfeghi and Hur [12] investigated the effect of SJ on S809 airfoil numerically using Detached-Eddy Simulation (DES) turbulence model. At a small angle of attack, the jet was subject to early separation and the lift coefficient was reduced. For the separated flow, the enhancement of the aerodynamic coefficients was observed. Zhao and Zhao [13] numerically investigated flow control around an OA213 rotor with a jet. Furthermore, focusing on a wind tunnel, Tang et al. [14] investigated the effect of the SJ on the low-speed airfoil. They implemented SJ in their proposed model in which the maximum lift coefficient was increased by $27.4 \%$ and the drag coefficient was decreased by $19.6 \%$. In addition, Giorgi et al. [15] compared and analyzed the effect of using two different flow control methods, namely SJ and Continuous Jet (CJ), on the boundary layer separation on a NACA 0015 airfoil. They concluded that the Synthetic Jet Actuator (SJA) was more useful in terms of regaining energy. Abe et al. [16] also applied the large-eddy method to conduct a simulation of installing a SJ at the leading edge on NACA 0015 airfoils and investigated the effects of actuation frequency. Neve et al. [17] also carried out parametric analysis to investigate the effect of frequency, jet angle, and jet velocity on the NACA 0015 at Reynolds number of 896000 . The jet angle $\left(30^{\circ}-40^{\circ}\right)$, jet frequency $(100 \mathrm{~Hz})$, and nondimensional jet velocity (1.8-2.0) could significantly affect the performance. Parthasarathy and Das [18] analyzed the physics of the flow and controlled the separated flow at $20^{\circ}$ angle of attack on the NACA 0015 airfoil at the Reynolds number of 896000 using S.J.

Both pitching airfoil and SJ mechanisms can be regarded as periodic functions. When these two mechanisms are simultaneously used, the phase difference between these two oscillations can affect the flow field. According to the previous research studies, the effect of phase difference has not been carefully investigated yet. In this study, an active flow control based on the SJ was applied to the NACA 0015 oscillating airfoil. The present study aims to investigate the effects of SJ on the dynamic stall control and characteristics of aerodynamic amelioration. Furthermore, the effect of phase difference between the airfoil and SJ oscillations on the aerodynamic characteristics was investigated. To this end, an in-house code based on the Reynoldsaveraged Navier-Stokes equations for the unsteady and turbulent flow was developed.

\section{Numerical methods}

\subsection{Governing equations}

The integral form of the two-dimensional compressible Navier-Stokes equations is described in the following [19]:

$$
\frac{\partial}{\partial t} \int_{\Omega} \vec{W} d \Omega+\oint_{\partial \Omega}\left(\vec{F}_{c}-V_{S} \vec{W}-\vec{F}_{v}\right) d S=0,
$$

where $\Omega, \partial \Omega$, and $V_{S}$ are the moving control volume, control surface, and control volume speed, respectively [20]. The following conservative variables, convection, and viscose flux are given below:

$$
\begin{aligned}
& \vec{W}=\left[\begin{array}{c}
\rho \\
\rho u \\
\rho v \\
\rho E
\end{array}\right], \quad \vec{F}_{c}=\left[\begin{array}{c}
\rho V_{r} \\
\rho u V_{r}+n_{x} p \\
\rho v V_{r}+n_{y} p \\
\rho H V_{r}+V_{S} p
\end{array}\right], \\
& \vec{F}_{v}=\left[\begin{array}{c}
0 \\
n_{x} \tau_{x x}+n_{y} \tau_{x y} \\
n_{x} \tau_{y x}+n_{y} \tau_{y y} \\
n_{x} \Theta_{x}+n_{y} \Theta_{y}
\end{array}\right]
\end{aligned}
$$

where $V_{r}$ is the relative velocity of the motion of flow and system [21]. The static pressure $(p)$ is also written as follows:

$$
\begin{aligned}
& p=\rho(\gamma-1)\left[E-\left(\frac{u^{2}+v^{2}}{2}\right)\right], \\
& p=\rho(\gamma-1)\left[E-\left(\frac{u^{2}+v^{2}}{2}\right)\right],
\end{aligned}
$$


where $E$ is the total energy per unit mass [22]. The shear stress components as well as $\Theta_{x}$ and $\Theta_{y}$ are expressed in [19]. This study investigates unsteady and turbulent flow of the airfoil at the chord Reynolds number (Reynolds number based on the airfoil chord length) of $10^{6}$. The chord Reynolds number is defined as follows:

$$
\operatorname{Re}=\frac{U_{\infty} c}{v}
$$

where $U_{\infty}$ is the free-stream velocity, $c$ is the chord length, and $v$ is the kinematic viscosity of the fluid [23]. In order to simulate the turbulent flow of interest, the widely known $k-k_{L}-\omega$ model [24] was employed. The abovementioned model consists of three transport equations and three transport equations including the turbulent kinetic energy $k_{T}$, laminar kinetic energy $k_{L}$, and specific dissipation rate $\omega$ [24].

For the time discretization of Eq. (1), an explicit scheme, as expressed in Eq. (5), was utilized. The fourth-order Runge-Kutta method was used to solve Eq. (5):

$$
\vec{W}^{n+1}-\vec{W}^{n}=-\frac{\Delta t}{\Omega} \vec{R}^{n}
$$

where $\Delta t, n$, and $\vec{R}$ are the global physical time step, time level, and residual vector, respectively [20]. In order to discretize the residual vector, a finite-volume scheme based on the second-order approximate Roe was employed [25]. On a structured grid, $\Delta t$ for a control volume $\Omega$ could be obtained through the following approximate relation [19]:

$$
\Delta t_{I}=C F L \frac{\Omega_{I}}{\left(\hat{\Lambda}_{c}^{I}+\hat{\Lambda}_{c}^{J}\right)_{I}}
$$

where the spectral radii of the convective flux Jacobeans are written as follows:

$$
\begin{aligned}
& \hat{\Lambda}_{c}^{I}=\left(\left|V^{I}+a\right| \Delta S^{I}\right), \\
& \hat{\Lambda}_{c}^{J}=\left(\left|V^{J}+a\right| \Delta S^{J}\right) .
\end{aligned}
$$

After conducting the time-step independency study, a minimum $\Delta t_{I}$ over all control volumes was selected to achieve time accuracy.

The following equation is used to simulate the motion of oscillating airfoil [26]:

$$
\alpha(t)=\alpha_{m}+\alpha_{0} \sin (\omega t)
$$

where $\alpha_{m}, \alpha_{0}$, and $\omega$ are the main angle of attack, angular amplitude, and angular frequency, respectively. The angular frequency depends on the reduced frequency and is defined as follows:

$$
k=\omega c / 2 U_{\infty} .
$$

\subsection{Grid generation and boundary conditions}

A C-type grid around the NACA 0015 airfoil is generated. This grid is archived with a combination of an o-type grid in the upstream zone and h-type grid in the downstream zone. By utilizing a C-type structure, a proper orthogonal grid, particularly near the leading and trailing edges, can be generated, as shown in Figure 1 [27].

In order to simulate the grid motion, the coordinate system origin was fixed on the one-quarter of the airfoil chord from the leading edge and the airfoil along with the computational domain oscillates around this point. Figure 2 shows the computational domain and applied boundary conditions. The grid domain includes a velocity inlet boundary, a pressure outlet boundary, and a solid wall (airfoil surface). The velocity of the SJ is described as follows [5]:

$$
u_{j}=U_{j} \sin (f . t+\varphi),
$$

where $U_{j}, \varphi$, and $f$ are the jet velocity amplitude, phase difference between the airfoil and jet, and non-dimensional frequency, respectively. The nondimensional frequency was expressed using the following equation [13]:

$$
f=\frac{\omega_{j} c}{2 U_{\infty}},
$$

where $\omega_{j}$ is the oscillation frequency of the SJ. The

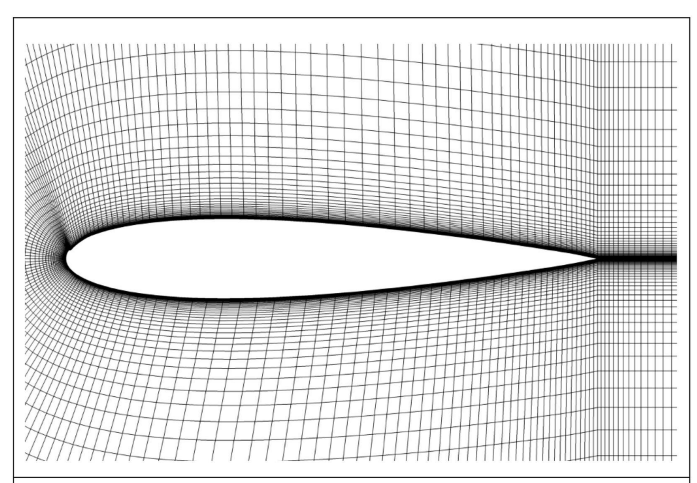

Figure 1. A part of the grid used in flow computations.

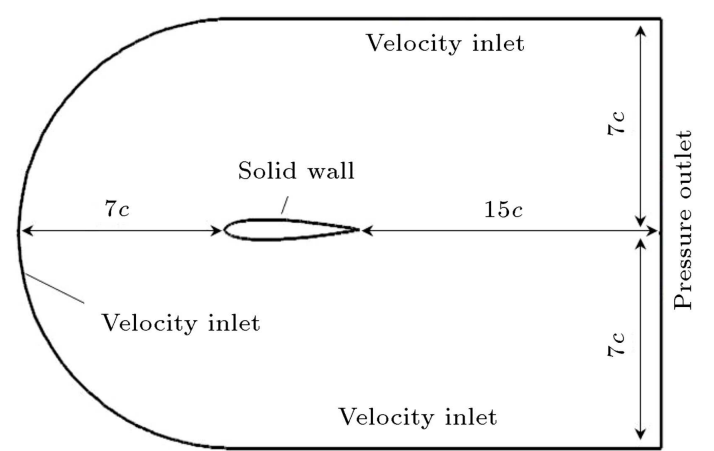

Figure 2. Computational domain and applied boundary conditions around NACA 0015 airfoil. 
ratio between the momentum of the free stream and the momentum of the jet is defined as the momentum coefficient $\left(c_{\mu}\right)$, as shown in the following [13]:

$$
c_{\mu}=C_{\mu} \sin (f . t+\varphi) ; \quad C_{\mu}=\frac{\rho_{j} U_{j}^{2} h}{1 / 2 \rho_{\infty} U_{\infty}^{2} c}
$$

where $C_{\mu}$ is the amplitude of the SJ and $h$ is the SJ throat width.

\section{Results}

\subsection{Grid independence and validation}

In order to evaluate the effects of the grid size on the results obtained from the numerical solutions, three grids including $285 \times 51,316 \times 61$, and $351 \times 66$ cells were tested. Figure 3 shows the lift coefficients of these three grids. As observed, the results of grids 2 and 3 are very close to each other. Due to a large amount of the computational cost of an unsteady solution, grid 2 is used for the subsequent computations. The first cell size of the selected grid is set so that $y^{+}<1$.

In order to evaluate the precision of the developed computer program, the present results were compared with those of previous studies on the lift and drag coefficients. First, the ability of the flow solver was tested for an oscillating airfoil case without SJ. To this end, the flow parameters $C_{l}$ and $C_{d}$ of the present study and the experimental results obtained by Piziali [28] were compared. A comparison was made in terms of a NACA 0015 airfoil at the Reynolds number of $1.935 \times$ $10^{6}$ with $\mathrm{Ma}=0.289$ and $k=0.134$ in the of angle of attack range of $17^{\circ} \pm 4^{\circ}$. The results of these comparisons are shown in Figures $4-6$. The anticipations are in close agreement with the experimental data. After evaluating the flow solver for the baseline airfoil without SJ, the lift coefficients of the stationary airfoil with the SJ of the present flow solver were compared with numerical results of Duvigneau and Visonneau [5]. This comparison was made concerning a NACA 0015

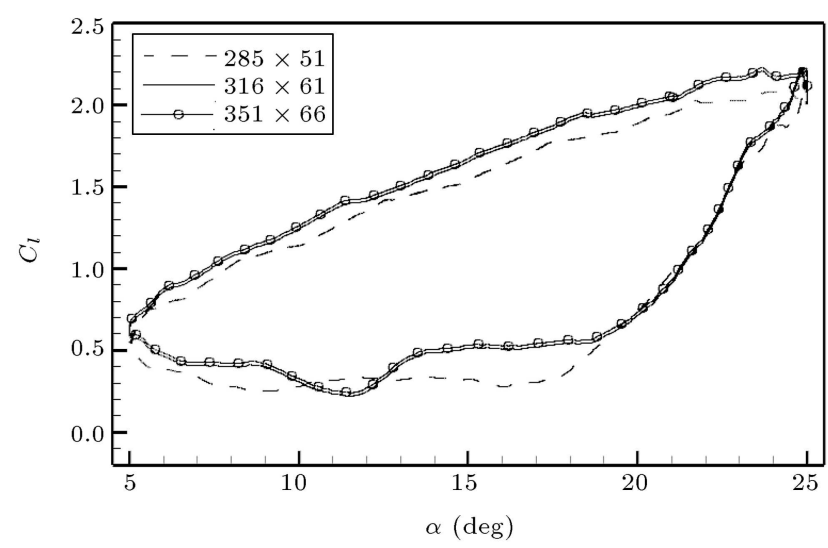

Figure 3. Comparison of the lift coefficients in terms of three computational grids based on the angle of attack.



Figure 4. Comparison of the present solver and experimental data [28] regarding the lift coefficients and angle of attack.

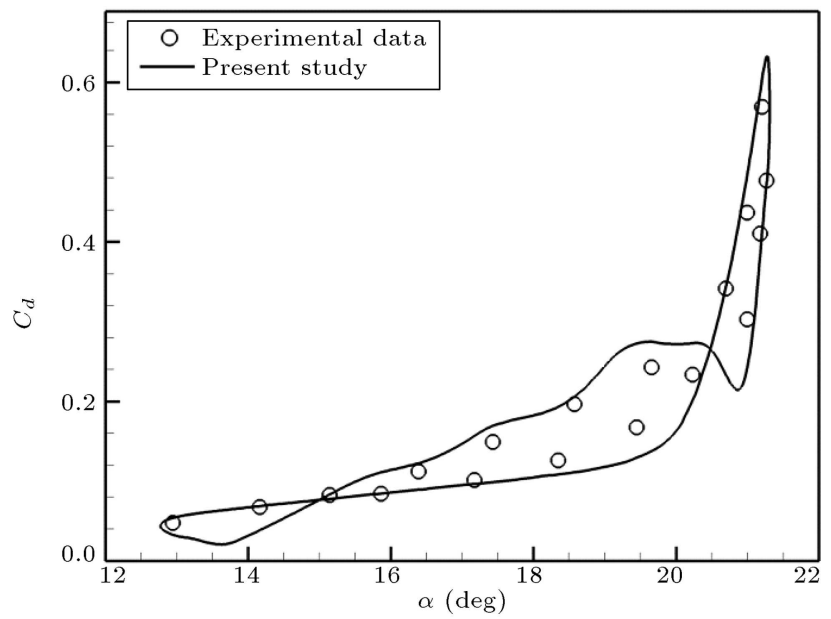

Figure 5. Comparison of the present solver and experimental data [28] regarding the drag coefficients and angle of attack.

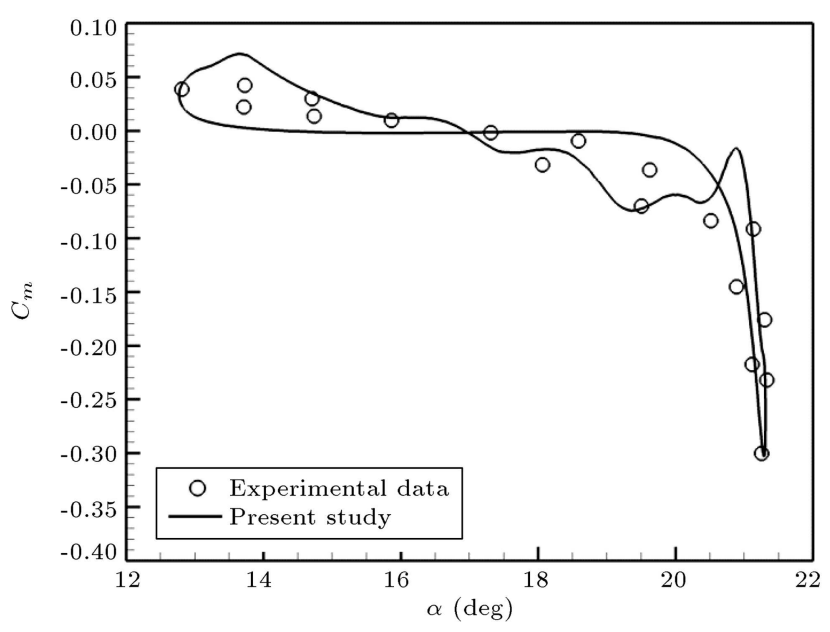

Figure 6. Comparison of the present solver and experimental data [28] regarding the pitching moment coefficients and angle of attack. 




Figure 7. Comparison of the present solver and experimental data [5] regarding the lift coefficients and angle of attack.

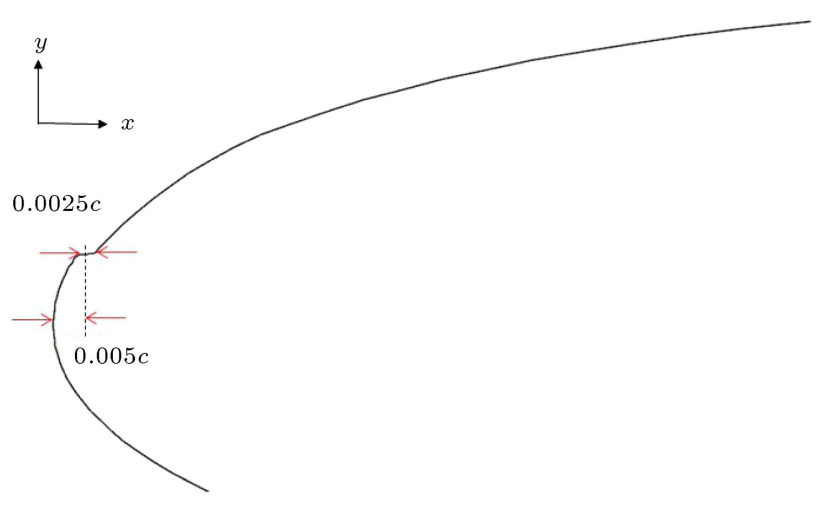

Figure 8. The situation of the Synthetic Jet (SJ) on the leading edge of airfoil.

airfoil at the Reynolds number of 896000 with $\mathrm{Ma}=$ 0.1. Figure 7 compares the results of the present solver with those of Duvigneau and Visonneau [5] for the lift coefficients with respect to the angle of attack.

\subsection{Simulating the SJ on the airfoil}

In this study, the slot of the tangential SJ was placed on the upper surface of the NACA 0015 airfoil centering at $0.5 \%$ chord with a height of $0.25 \%$ chord. Figure 8 shows the situation of the S.J on the airfoil leading edge. The investigation was conducted at $\mathrm{Ma}=0.2$ and $\operatorname{Re}=10^{6}$. In the following section, the effects of the investigated parameters of the SJ on the aerodynamic coefficients are studied. These parameters include the magnitude of momentum coefficient, reduced frequency, and phase difference.

\subsection{The effect of momentum coefficient}

The current study aims to explore the changes in the aerodynamic characteristics with variations in the jet momentum coefficient. To this end, a comparison was made to examine the three jet momentum coefficients including $C_{\mu}=0.07,0.1$, and 0.13 . The results of the comparison of these three SJ control cases are

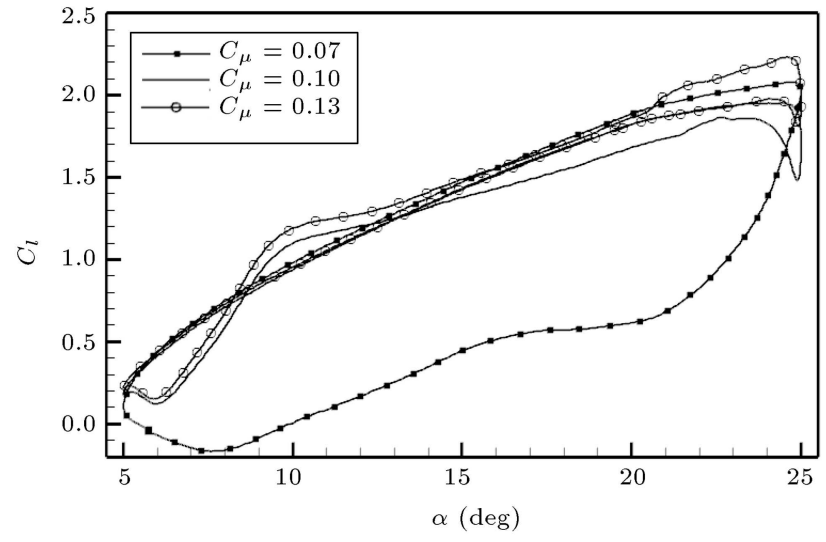

Figure 9. Comparison of the lift coefficients with respect to $\alpha$ for Synthetic Jet (SJ) with different momentum coefficients.

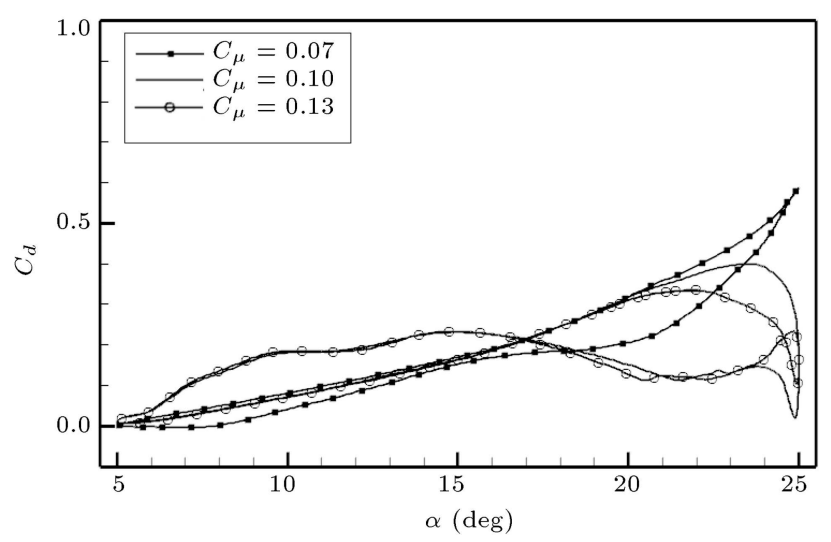

Figure 10. Comparison of the drag coefficients with respect to $\alpha$ for Synthetic Jet (SJ) with different momentum coefficients.

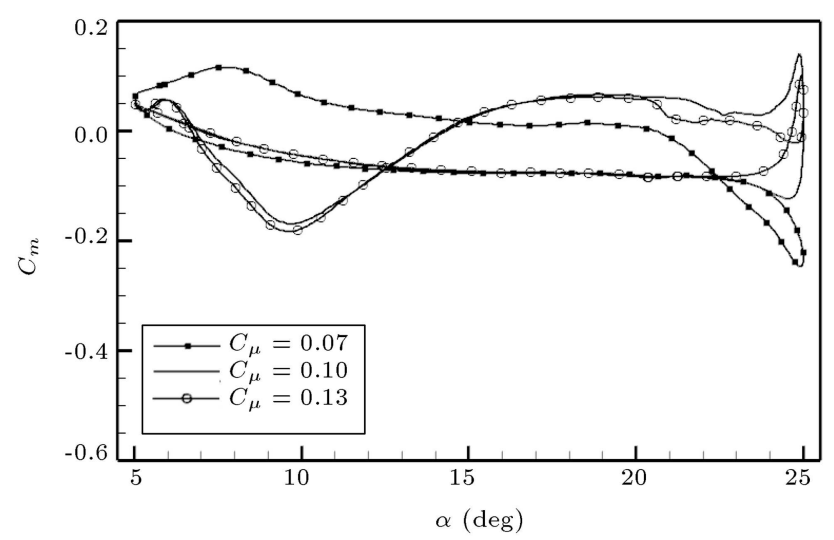

Figure 11. Comparison of the drag coefficients with respect to $\alpha$ for Synthetic Jet (SJ) with different pitching moment coefficients.

shown in Figures 9-11. These investigations were performed at the reduced frequency of $k=0.25$ and $f=k$. The results also indicated that SJ with the higher momentum coefficient $\left(C_{\mu}=0.13\right)$ had a better ability in lift enhancement. In addition, by increasing 
the jet momentum coefficient, the hysteresis loops of aerodynamic coefficients became thinner. Figure 10 reveals that the SJ control case with $C_{\mu}=0.07$ has the maximum drag coefficient at $25^{\circ}$ and the aerodynamic coefficient loops indicate larger hysteresis in the lift coefficient curve than in other cases. According to the findings, by increasing the jet momentum, more energy can be transferred to the boundary layer. Consequently, further improvements were carried out to increase the lift and reduce the drag coefficients. For the case with $C_{\mu}=0.1,0.13$, the lift coefficient curves do not show significant stall, indicating that a stronger jet is able to completely control the dynamic stall. In order to quantify the improvement of the results of SJ control for enhancing the lift coefficient and the decrease in the drag and pitching moment coefficients, the differences in the area under the $C_{l}, C_{d}$, and $C_{m}$ curves among the control cases and the baseline airfoils were calculated [29] as follows (where $q$ is either drag or moment):

$$
\Delta A_{C q}=\frac{\int_{0}^{2 \pi}\left(C_{q}^{\text {baseline }}-C_{q}^{\text {control }}\right) d \alpha}{\int_{0}^{2 \pi} C_{q}^{\text {baseline }} d \alpha} .
$$

A summary of the obtained values for SJ control is given in Table 1. It reveals that by implementing the SJ control with a sufficient momentum coefficient, the amplitude of lift coefficients dramatically increases. Furthermore, the amplitudes of drag and moment coefficients are noticeably diminished. Increasing the lift and decreasing the drag can significantly improve the aerodynamic performance of the airfoil. For $C_{\mu}=$ 0.07 , the amplitudes of lift and drag can decrease by $17.76 \%$ and increase by $5 \%$, respectively. Consequently, this jet momentum coefficient does not show a better performance than the baseline. The amplitudes of lift can increase by $14.95 \%$ and $23.36 \%$ in the SJ control case with $C_{\mu}=0.1$ and 0.13 , respectively. The amplitudes of drag can decrease by as much as $22.5 \%$ and $25.5 \%$ with $C_{\mu}=0.1$ and 0.13 , respectively. Moreover, the amplitude of moment can be reduced by $55.81 \%$ and $53.49 \%$, as shown in Table 1 . Of note, the
SJ controls with $C_{\mu}=0.1$ and 0.13 are of identical ability to reduce drag and moment coefficients, i.e., in a jet with higher momentum, no further improvement in decreasing the amplitudes of drag and moment coefficients is observed; however, it may enhance the lift. With an increase in $C_{\mu}$ from 0.1 to 0.13 , the drag amplitude would reduce from $22.5 \%$ to $25.5 \%$. Moreover, the moment amplitude would decrease from $55.81 \%$ to $53.49 \%$. Generally, the findings of the present study are encouraging. In fact, the SJ control can significantly increase the lift and reduce the drag and pitching moment.

\subsection{The effect of phase difference at $k=0.25$ and $C_{\mu}=0.1$}

Another control parameter of the jet is responsible for investigating the aerodynamic characteristics of the NACA 0015 airfoil with many phase differences. Given that airfoil and jet oscillations are both sinusoidal, one can consider a phase difference between them to study this effect. In fact, if the oscillating airfoil is at the maximum angle of attack, the oscillating jet will have the highest jet velocity; thus, the phase difference will be zero. Otherwise, there will be a phase difference between these oscillations. Figures 1223 show the modifications of lift, drag, and pitching moment coefficients compared to the baseline under four phase differences. In all these cases, the same jet

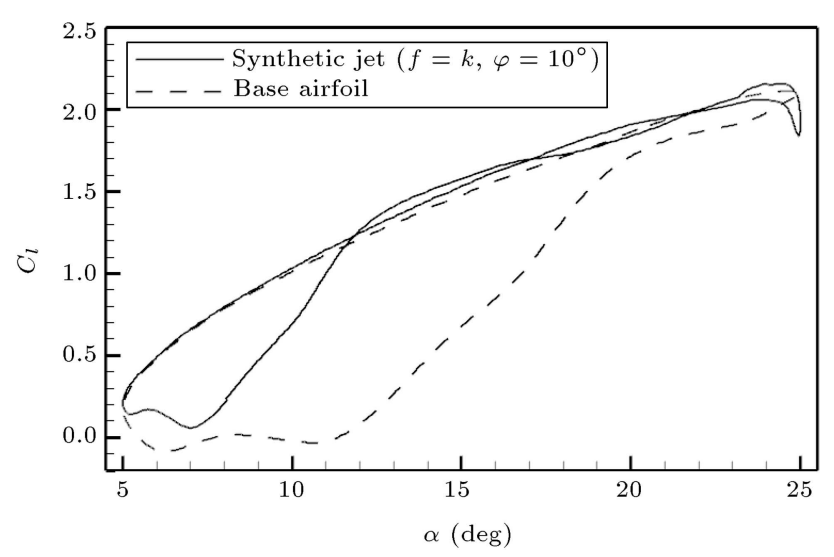

Figure 12. Comparison of the lift coefficients with respect to $\alpha$ for Synthetic Jet (SJ) with $\varphi=10^{\circ}$.

Table 1. Comparison of the effects of the synthetic jet cases and their baseline airfoils $(k=0.25)$.

\begin{tabular}{ccccc}
\hline Coefficient & Baseline & $\boldsymbol{C}_{\boldsymbol{\mu}}=\mathbf{0 . 0 7}$ & $\boldsymbol{C}_{\boldsymbol{\mu}}=\mathbf{0 . 1}$ & $\boldsymbol{C}_{\boldsymbol{\mu}}=\mathbf{0 . 1 3}$ \\
\hline$C_{l, a m p}$ & 1.07 & 0.88 & 1.23 & 1.32 \\
$C_{d, a m p}$ & 0.2 & 0.21 & 0.155 & 0.149 \\
$C_{m, a m p}$ & -0.043 & -0.037 & -0.019 & -0.02 \\
$\Delta A_{C_{l}}$ & - & $17.76 \% \downarrow$ & $14.95 \% \uparrow$ & $23.36 \% \uparrow$ \\
$\Delta A_{C_{d}}$ & - & $5 \% \uparrow$ & $22.5 \% \downarrow$ & $25.5 \% \downarrow$ \\
$\Delta A_{C_{m}}$ & - & $13.95 \% \downarrow$ & $55.81 \% \downarrow$ & $53.49 \% \downarrow$ \\
\hline
\end{tabular}




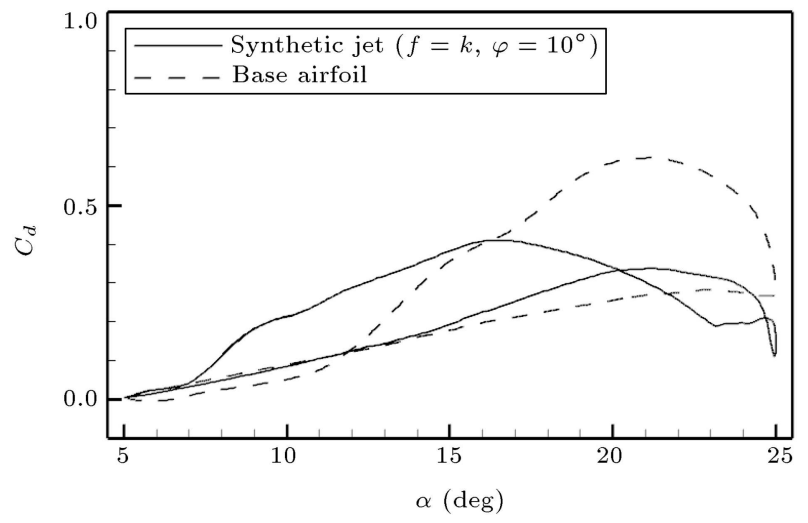

Figure 13. Comparison of the drag coefficients with respect to $\alpha$ for Synthetic Jet (SJ) with $\varphi=10^{\circ}$.

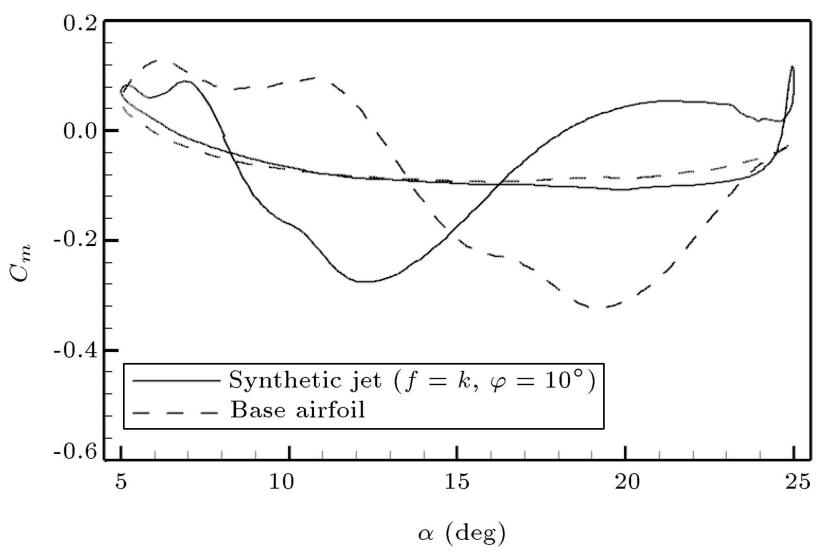

Figure 14. Comparison of the pitching moment coefficients with respect to $\alpha$ for Synthetic Jet (SJ) with $\varphi=10^{\circ}$.

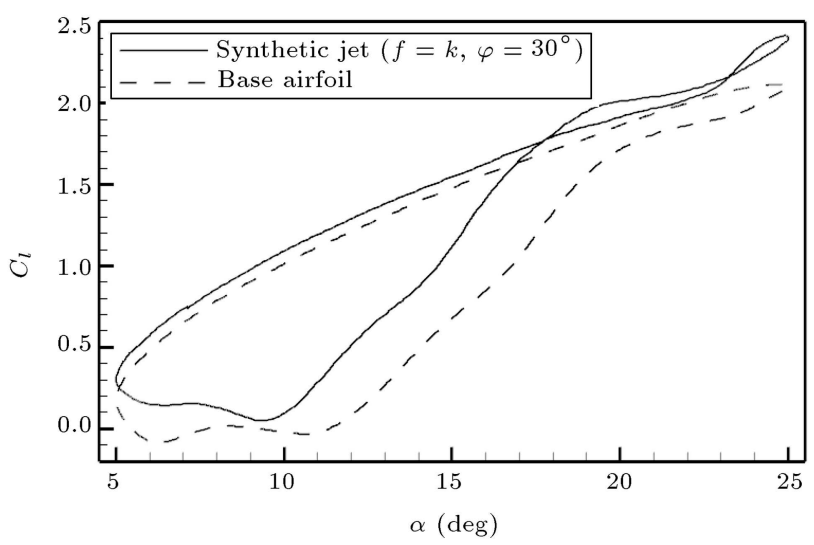

Figure 15. Comparison of the lift coefficients with respect to $\alpha$ for Synthetic Jet (SJ) with $\varphi=30^{\circ}$.

momentum $C_{\mu}=0.1$ with the reduced frequency of $k=$ 0.25 and $f=k$ was considered. Similar to the previous section, to quantify the enhancement in the lift and the decrease in the drag and pitching moment during a pitch cycle, the differences of the area under the $C_{l}$, $C_{d}$, and $C_{m}$ curves among the S.J control cases and their baseline airfoils were estimated. Table 2 shows the

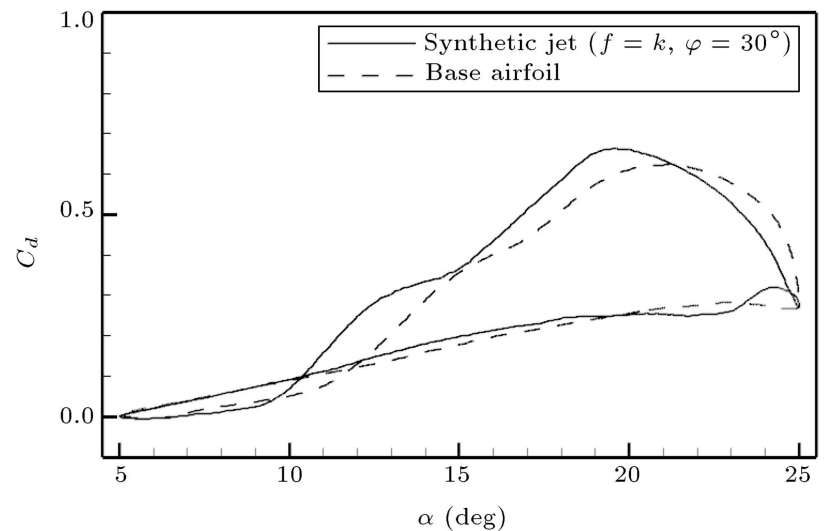

Figure 16. Comparison of the drag coefficients with respect to $\alpha$ for Synthetic Jet (SJ) with $\varphi=30^{\circ}$.

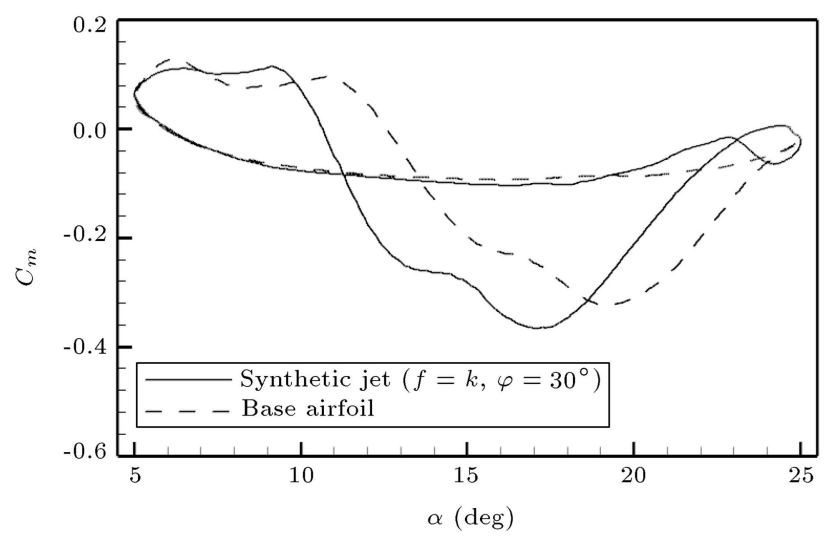

Figure 17. Comparison of the pitching moment coefficients with respect to $\alpha$ for Synthetic Jet (SJ) with $\varphi=30^{\circ}$.

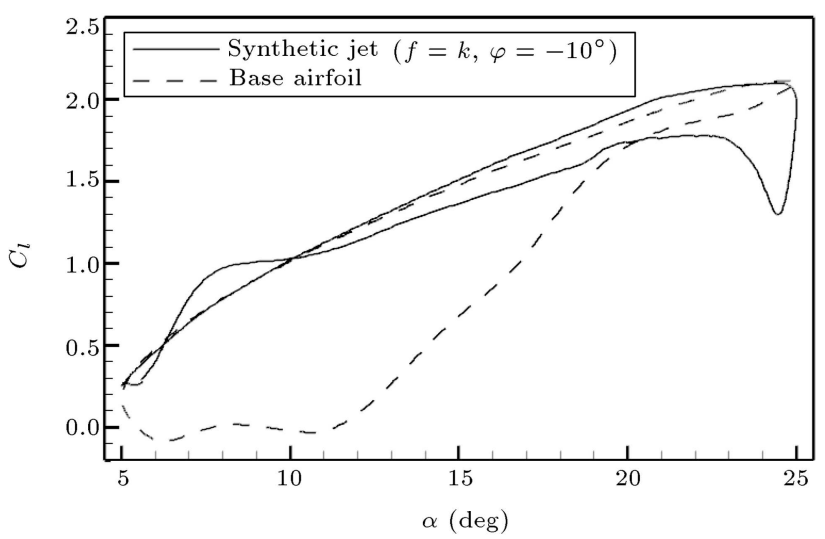

Figure 18. Comparison of the lift coefficients with respect to $\alpha$ for Synthetic Jet (SJ) with $\varphi=-10^{\circ}$.

modifications of the aerodynamic characteristics with many phase differences. As observed, with $\varphi=-30^{\circ}$, the lift would increase by $20.56 \%$ and with $\varphi=-10^{\circ}$, the drag and pitching moment are reduced by $25.5 \%$ and $60.46 \%$, respectively. When the phase differences vary from $-10^{\circ}$ to $-30^{\circ}$, the amount of amplitude drag and pitching moment would reduce from $25.5 \%$ to 




Figure 19. Comparison of the drag coefficients with respect to $\alpha$ for Synthetic Jet (SJ) with $\varphi=-10^{\circ}$.

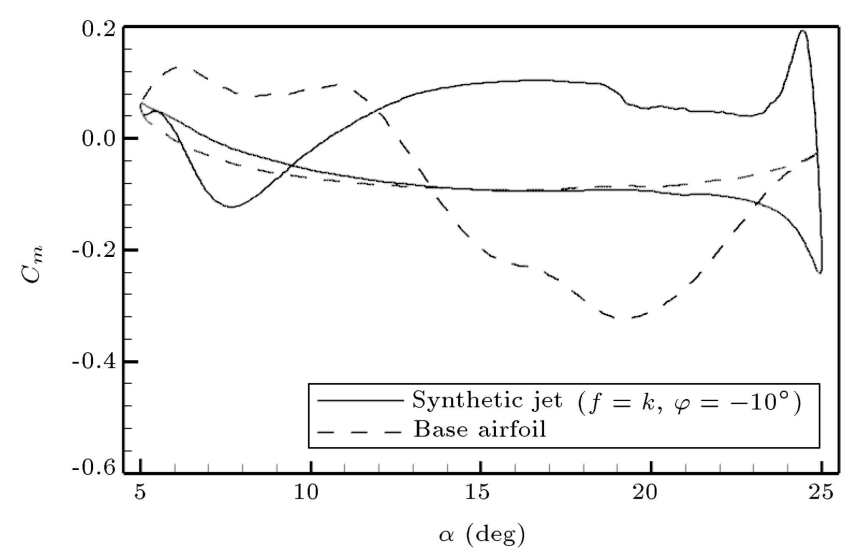

Figure 20. Comparison of the pitching moment coefficients with respect to $\alpha$ for Synthetic Jet (SJ) with $\varphi=-10^{\circ}$.

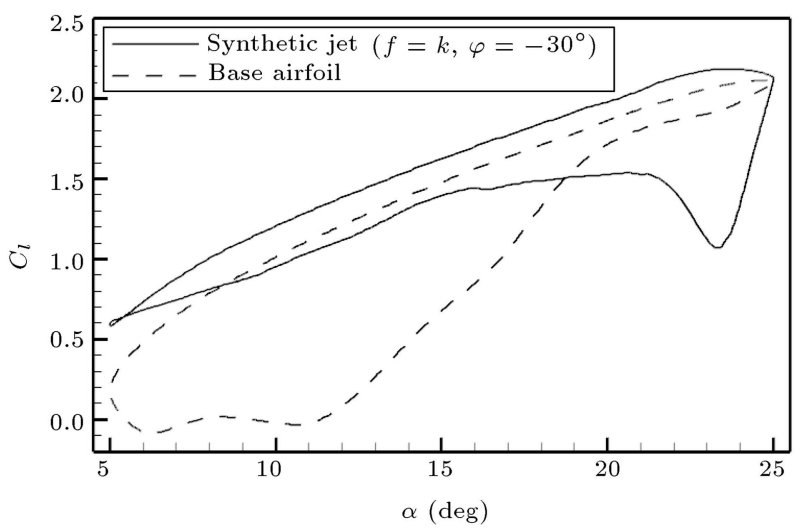

Figure 21. Comparison of the lift coefficients with respect to $\alpha$ for Synthetic Jet (SJ) with $\varphi=-30^{\circ}$.

$25 \%$ and decrease from $60.46 \%$ to $41.86 \%$, respectively. The results indicated that the SJ control with $\varphi=$ $-30^{\circ}$ had the highest amplitude of lift. In order to compare the drag and pitching moment coefficients, the multiplication of $\Delta A_{C_{d}}$ and $\Delta A_{C_{m}}$ was calculated. The results of the multiplication in Table 2 indicate that the

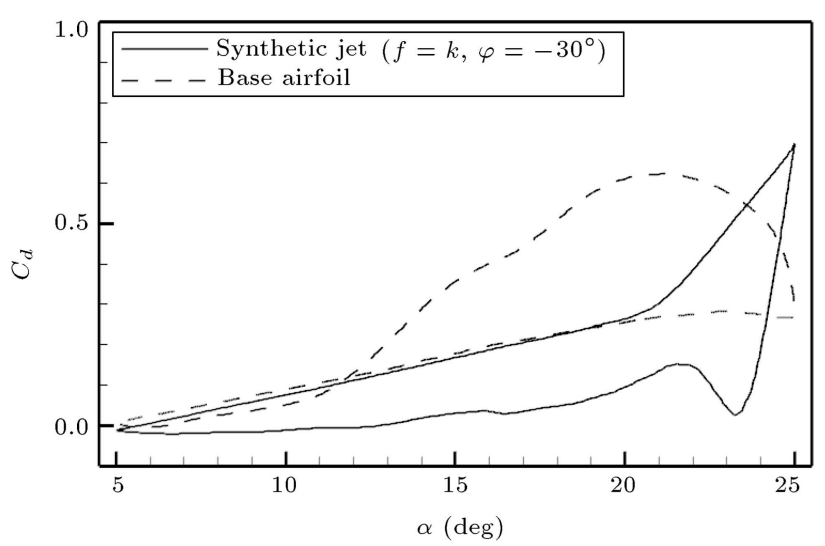

Figure 22. Comparison of the drag coefficients with respect to $\alpha$ for Synthetic Jet (SJ) with $\varphi=-30^{\circ}$.

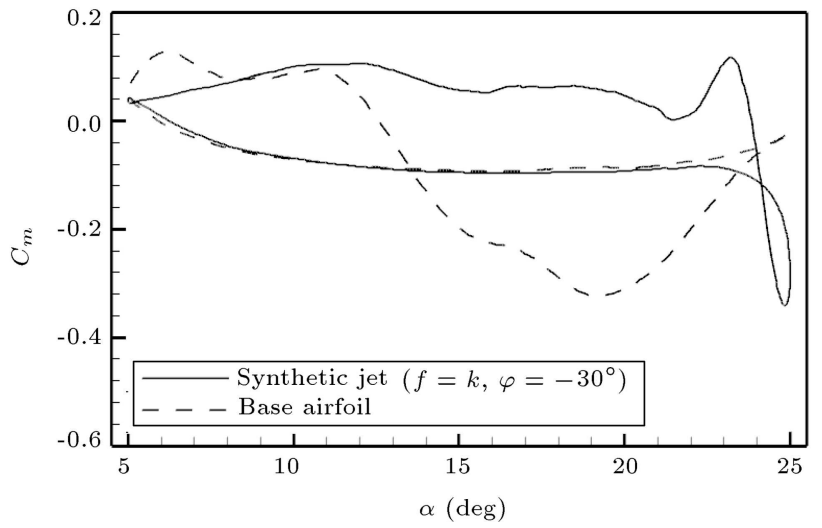

Figure 23. Comparison of the pitching moment coefficients with respect to $\alpha$ for Synthetic Jet (SJ) with $\varphi=-30^{\circ}$.

SJ control case with $\varphi=30^{\circ}$ does not outperform the baseline because the multiplication of the amplitudes drag and moment coefficients shows a 5.8 increase with respect to the baseline. Moreover, the multiplication of the amplitudes of drag and moment coefficients in the SJ control case with $\varphi=-10^{\circ}$ ensures the highest improvement.

\subsection{The effect of phase difference at $k=0.15$ and $C_{\mu}=0.1$}

This section aims to investigate the effects of SJ control with a reduced frequency of $k=0.15$ under two phase differences $\left(\varphi=0^{\circ}\right.$ and $\left.\varphi=-30^{\circ}\right)$. Figures 2429 show the results obtained from comparing the SJ cases and their baselines regarding the lift, drag, and pitching moment coefficients. Table 3 presents the amplitudes of the aerodynamic coefficients of the many phase differences and their enhancement compared to the baselines. It also indicates that in the SJ control case with $\varphi=-30^{\circ}$, the amplitude of lift would increase by $27.98 \%$. Furthermore, the amplitude of drag and pitching moment would reduce by $14.98 \%$ and $23.08 \%$, respectively. The results indicate that at the 
Table 2. Comparison of the effects of the SJ actuation with $C_{\mu}=0.1$ and $k=0.25$ and the baseline.

\begin{tabular}{ccccccc}
\hline Coefficient & Baseline & $\boldsymbol{\varphi}=\mathbf{0}^{\circ}$ & $\boldsymbol{\varphi}=\mathbf{1 0}^{\circ}$ & $\boldsymbol{\varphi}=\mathbf{3 0}^{\circ}$ & $\boldsymbol{\varphi}=\mathbf{- 1 0 ^ { \circ }}$ & $\boldsymbol{\varphi}=\mathbf{- 3 0 ^ { \circ }}$ \\
\hline$C_{l, a m p}$ & 1.07 & 1.23 & 1.236 & 1.25 & 1.22 & 1.29 \\
$C_{d, a m p}$ & 0.2 & 0.155 & 0.169 & 0.205 & 0.149 & 0.15 \\
$C_{m, a m p}$ & -0.043 & -0.019 & -0.027 & -0.044 & -0.017 & -0.025 \\
$\Delta A_{C_{l}}$ & - & $14.95 \% \uparrow$ & $15.51 \% \uparrow$ & $16.82 \% \uparrow$ & $14.01 \% \uparrow$ & $20.56 \% \uparrow$ \\
$\Delta A_{C_{d}}$ & - & $22.5 \% \downarrow$ & $15.5 \% \downarrow$ & $2.5 \% \uparrow$ & $25.5 \% \downarrow$ & $25 \% \downarrow$ \\
$\Delta A_{C_{m}}$ & - & $55.81 \% \downarrow$ & $37.21 \% \downarrow$ & $2.32 \% \uparrow$ & $60.46 \% \downarrow$ & $41.86 \% \downarrow$ \\
$\Delta A_{C_{d}} \times \Delta A_{C_{m}}$ & - & $1255.7 \downarrow$ & $576.75 \downarrow$ & $5.8 \uparrow$ & $1541.7 \downarrow$ & $1046.5 \downarrow$ \\
\hline
\end{tabular}

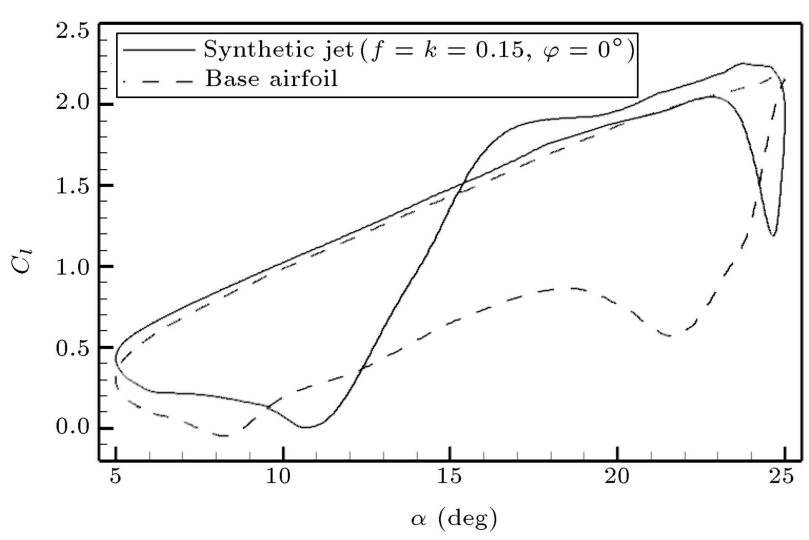

Figure 24. Comparison the lift coefficients with respect to $\alpha$ for Synthetic Jet (SJ) with $\varphi=0^{\circ}$.

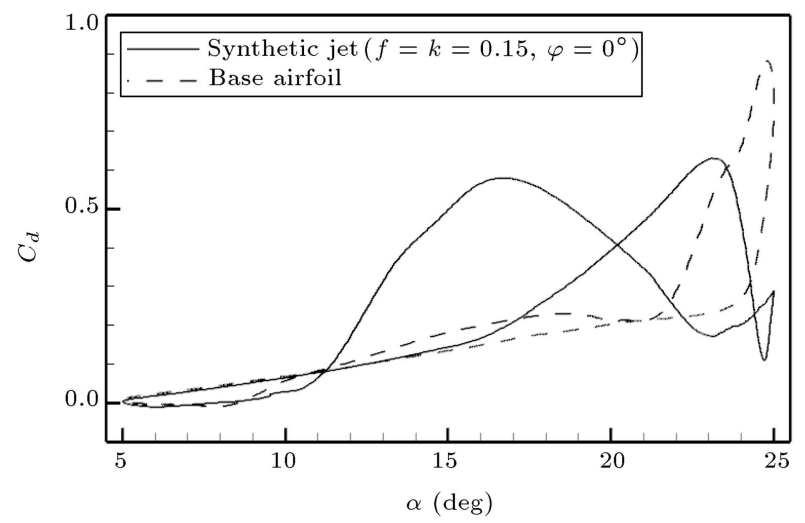

Figure 25. Comparison the drag coefficients with respect to $\alpha$ for Synthetic Jet (SJ) with $\varphi=0^{\circ}$.

$-30^{\circ}$ phase, i.e., the difference between the phases of the airfoil and jet, the amplitudes of drag and moment coefficient were reduced significantly more than those at the phase $0^{\circ}$. However, the lift coefficient did not considerably increase compared to that at the phase $0^{\circ}$.

\section{Conclusions}

In the present study, the dynamic stall control was numerically investigated using a Synthetic Jet (SJ) on the NACA 0015 at $\operatorname{Re}=10^{6}$. The solver was validated

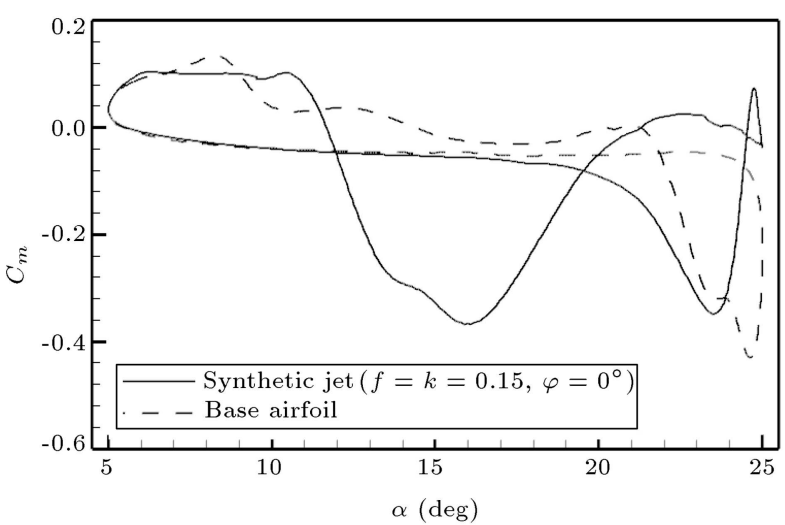

Figure 26. Comparison the pitching moment coefficients with respect to $\alpha$ for Synthetic Jet (SJ) with $\varphi=0^{\circ}$.

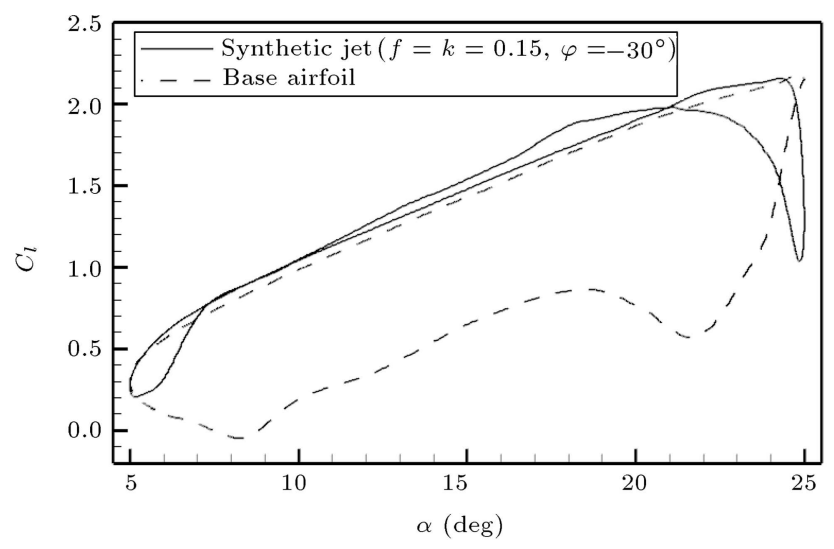

Figure 27. Comparison the lift coefficients with respect to $\alpha$ for Synthetic Jet (SJ) with $\varphi=-30^{\circ}$.

against the results of the baseline experiment regarding the oscillating airfoil. The comparison of the results of SJ control and those of the numerical investigation of the static airfoil showed good agreement. First, the effects of varying the jet momentum coefficients on the dynamic stall control performance were investigated. The results indicated that using the SJ control with proper momentum coefficient could considerably control the separation. As a result, the dynamic stall was delayed or arrested. Three momentum coefficients of $0.07,0.1$, and 0.13 were investigated. The SJ cases 
Table 3. Comparison of the SJ cases with the baseline.

\begin{tabular}{cccc}
\hline Coefficient & Baseline & $\boldsymbol{\varphi}=\mathbf{0}^{\circ}$ & $\boldsymbol{\varphi}=-\mathbf{3 0}^{\circ}$ \\
\hline$C_{l, a m p}$ & 0.965 & 1.18 & 1.235 \\
$C_{d, a m p}$ & 0.188 & 0.186 & 0.16 \\
$C_{m, a m p}$ & -0.039 & -0.042 & -0.03 \\
$\Delta A_{C_{l}}$ & - & $22.28 \% \uparrow$ & $27.98 \uparrow$ \\
$\Delta A_{C_{d}}$ & - & $1.06 \% \downarrow$ & $14.89 \% \downarrow$ \\
$\Delta A_{C_{m}}$ & - & $7.69 \% \uparrow$ & $23.08 \% \downarrow$ \\
\hline
\end{tabular}

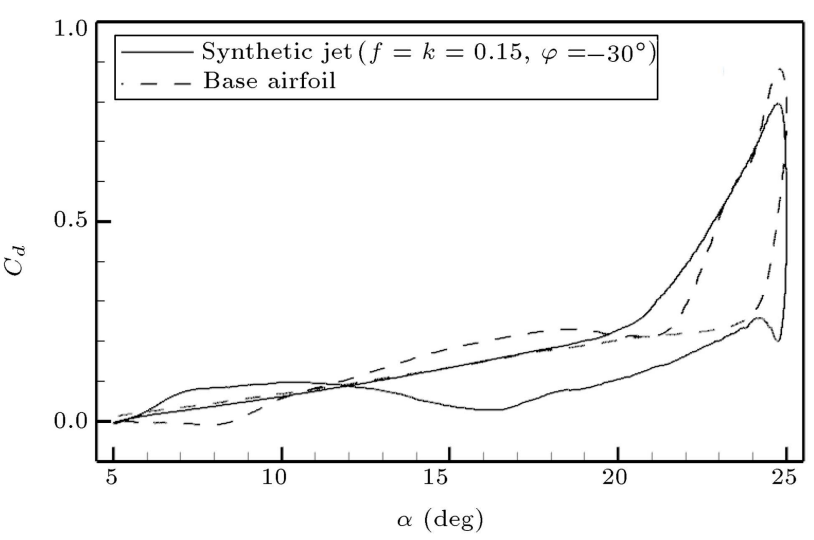

Figure 28. Comparison the drag coefficients with respect to $\alpha$ for Synthetic Jet (SJ) with $\varphi=-30^{\circ}$.

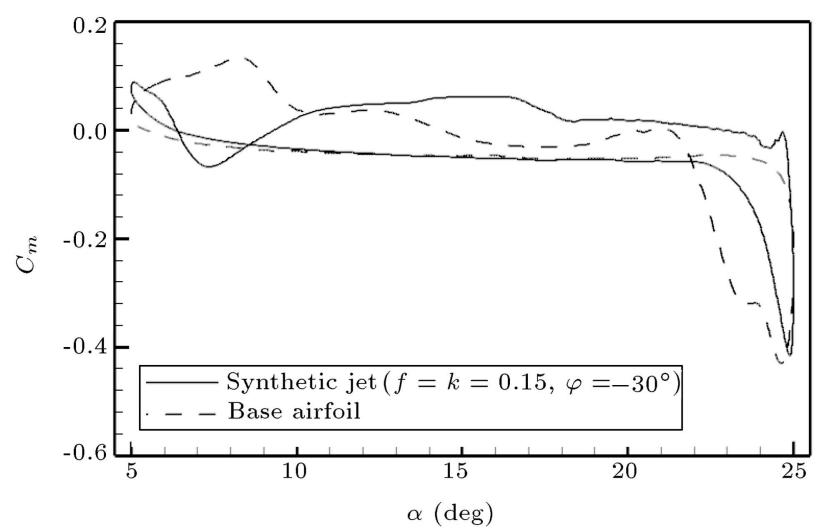

Figure 29. Comparison the pitching moment coefficients with respect to $\alpha$ for Synthetic Jet (SJ) with $\varphi=-30^{\circ}$.

with the momentum coefficients of $C_{\mu}=0.1$ and 0.13 exhibited an acceptable performance in eliminating the dynamic stall onset. The lower momentum of the jet required lower energy consumption. Therefore, the jet with the momentum coefficient of 0.1 was utilized to continue the investigation. The oscillations of the airfoil and jet were both sinusoidal. Thus, the effect of phase difference among them might be considerable. This effect has not been addressed in previous studies. To this end, in order to evaluate the effect of phase difference between the jet and airfoil, a range of phase differences between $-30^{\circ}$ and $+30^{\circ}$ were studied. Furthermore, the effect of the SJ at two different reduced frequencies was investigated. The conclusions and improvements of this research versus the baseline airfoils are given below:

1. For the case with the reduced frequency of $k=0.25$ and phase difference of $\varphi=0^{\circ}$, the amplitude of lift could increase by $14.95 \%$ and the amplitude of drag and pitching moment could decrease by $22.5 \%$ and $55.81 \%$, respectively;

2. The results showed that the case with the reduced frequency of $k=0.25$ had the highest amplitude of lift at $\varphi=-30^{\circ}$, which was improved by $20.56 \%$. Furthermore, at $\varphi=-10^{\circ}$, the drag amplitude and pitching moment coefficients were reduced by $25.5 \%$ and $60.46 \%$, respectively, which presented the best performance and a considerable decrease;

3. For the case with a lower reduced frequency of $k=0.15$, the best aerodynamic performances were achieved at $\varphi=-30^{\circ}$. In this phase difference, the amplitude of lift was increased by $27.98 \%$. In addition, the amplitude of drag and the amplitude of pitching moment were reduced by $14.98 \%$ and $23.08 \%$, respectively.

\section{Nomenclature}

$\begin{array}{ll}a & \text { Speed of sound } \\ c & \text { Airfoil chord } \\ C_{l} & \text { Lift coefficient } \\ C_{d} & \text { Drag coefficient } \\ C_{m} & \text { Pitching moment coefficient } \\ k & \text { Reduced frequency } \\ f & \text { Synthetic jet forcing frequency } \\ \operatorname{Re} & \text { Reynolds number } \\ h & \text { Actuation surface } \\ \mathrm{Ma} & \text { Mach number } \\ C_{\mu} & \text { Momentum coefficient }\end{array}$

\section{Greek}

$\alpha \quad$ Angle of attack

$\mu \quad$ Dynamic viscosity

$\rho \quad$ Density

$\eta \quad$ Similarity variable

$\varphi \quad$ Phase difference

\section{Subscripts}

$\begin{array}{ll}\infty & \text { Free stream (far field) } \\ t & \text { Turbulent }\end{array}$

\section{References}

1. Zhao, Q., Ma, Y., and Zhao, G. "Parametric analyses on dynamic stall control of rotor airfoil via synthetic 
jet", Chinese Journal of Aeronautics, 30(6), pp. 18181834 (2017).

2. Xu, H., Qiao, C., and Ye, Z. "Dynamic stall control on the wind turbine airfoil via a co-flow jet", Energies, 9(6), pp. 429-454 (2016).

3. Pasandideh Fard, M. and Sahaf, S.A. "A novel method for maximum lift prediction in high-lift configurations", Scientia Iranica, 23(2), pp. 668-677 (2016).

4. Heydari, A., Pasandideh-Fard, M., and Malekjafarian, M. "Investigation of unsteady parameters effects on aerodynamic coefficients of pitching airfoil using coarse grid computational fluid dynamic", Scientia Iranica, 21(2), pp. 370-386 (2014).

5. Duvigneau, R. and Visonneau, M. "Optimization of a synthetic jet actuator for aerodynamic stall control", Computers \& Fluids, 35(6), pp. 624-638 (2006).

6. Esmaeili, H. Monir, H. Tadjfar, M., and Bakhtian, A. "Tangential synthetic jets for separation control", Journal of Fluids and Structures, 45, pp. 50-65 (2014).

7. Zhang, W., Zhang, Z., Chen, Z., and Tang, Q. "Main characteristics of suction control of flow separation of an airfoil at low Reynolds numbers", European Journal of Mechanics-B/Fluids, 65, pp. 88-97 (2017).

8. Tran, S.A., McGlynn, E., and Sahni, O. "Large eddy simulation of flow interactions of a finite-span synthetic jet on an airfoil", 55th AIAA Aerospace Sciences Meeting, pp. 1-11 (2017).

9. Montazer, E., Mirzaei, M., Salami, E., Ward, T.A., Romli, F.I., and Kazi, S.N. "Optimization of a synthetic jet actuator for flow control around an airfoil", IOP Conference Series: Materials Science and Engineering, 152, p. 012023 (2016).

10. Tran, S.A., Sahni, O., and Corson, D. "Synthetic jet based active flow control of dynamic stall phenomenon on wind turbines under yaw misalignment", in 32nd ASME Wind Energy Symposium, AIAA SciTech Forum, National Harbor, Maryland (2014).

11. Yousefi, K., Saleh, R., and Zahedi, P. "Numerical study of blowing and suction slot geometry optimization on NACA 0012 airfoil", Journal of Mechanical Science and Technology, 28(4), pp. 1297-1310 (2014).

12. Moshfeghi, M. and Hur, N. "Numerical study on the effects of a synthetic jet actuator on S809 airfoil aerodynamics at different flow regimes and jet flow angles", Journal of Mechanical Science and Technology, 31(3), pp. 1233-1240 (2017).

13. Zhao, G. and Zhao, Q. "Parametric analyses for synthetic jet control on separation and stall over rotor airfoil", Chinese Journal of Aeronautics, 27(5), pp. 1051-1061 (2014).

14. Tang, H., Salunkhe, P., Zheng, Y., Du, J., and Wu, Y. "On the use of synthetic jet actuator arrays for active flow separation control", Experimental Thermal and Fluid Science, 57, pp. 1-10 (2014).
15. De Giorgi, M.G., De Luca, C.G., Ficarella, A., and Marra, F. "Comparison between synthetic jets and continuous jets for active flow control: Application on a NACA 0015 and a compressor stator cascade", Aerospace Science and Technology, 43, pp. 256-280 (2015).

16. Abe, Y., Okada, K., Nonomura, T., and Fujii, K. "The effects of actuation frequency on the separation control over an airfoil using a synthetic jet", Progress in Flight Physics, 7, pp. 147-168 (2015).

17. Neve, M., Kalamkar, V.R., and Wagh, A. "Numerical analysis of NACA aerofoil using synthetic jet", V001T01A006 (2017).

18. Parthasarathy, T. and Das, S.P. "Some aspects of flow control over a NACA0015 airfoil using synthetic jets", Journal of Physics: Conference Series, 822, p. 012009 (2017).

19. Blazek, J., Computational Fluid Dynamics: Principles and Applications, Elsevier Science Ltd. (2001).

20. Salimipour, S.E., Teymourtash, A.R., and Mamourian, M. "Investigation and comparison of performance of some air gun projectiles with nose shape modifications", Proceedings of the Institution of Mechanical Engineers, Part P: Journal of Sports Engineering and Technology, 233(1), pp. 3-15 (2018).

21. Salimipour, S.E., Teymourtash, A.R., and Mamourian, M. "Trajectory modification of a transonic spherical projectile under Hop-up mechanism", Journal of Scientia Iranica, Transactions B: Mechanical Engineering, 26(2), pp. 796-807 (2019).

DOI: $10.24200 /$ SCI.2018.20224

22. Zhang, Z., Zhang, W., Chen, Z., Sun, X., and Xia, C. "Suction control of flow separation of a low-aspectratio wing at a low Reynolds number", Fluid Dynamics Research, 50(6), p. 065504 (2018).

23. Bachant, P. and Wosnik, M. "Effects of Reynolds number on the energy conversion and near-wake dynamics of a high solidity vertical-axis cross-flow turbine", Energies, 9(2), p. 73 (2016).

24. Salimipour, S.E. "A modification of the k-kL- $\omega$ turbulence model for simulation of short and long separation bubbles", Computers \& Fluids, 181, pp. 67-76 (2019).

25. Roe, P.L. "Approximate Riemann solvers, parameter vectors, and difference schemes", Journal of Computational Physics, 43(2), pp. 357-372 (1981).

26. Salimipour, S.E. and Yazdani, Sh. "Dynamic stall control of low Reynolds number airfoil with separation bubble control blade", Modares Mechanical Engineering, 15(6), pp. 393-401 (2015) (In Persian).

27. Latha, S. and Gayathri, R. "Comparison between algebraic grid and elliptic grid over an airfoil", International Journal of Advance Research In Science And Engineering, 4(03) (March 2015). 
28. Piziali, R.A. "2-D and 3-D oscillating wing aerodynamics for a range of angles of attack including stall", NASA Ames Research Center; Moffett Field, CA, United States (1994).

29. Tran, S.A. Fisher, A.E., Corson, D., and Sahni, O. "Dynamic stall alleviation for an SC1095 airfoil using synthetic jet actuation", 53rd AIAA Aerospace Sciences Meeting, 5-9 January 2015, Kissimmee, Florida (2015).

\section{Biographies}

Ali Shokrgozar Abbasi, born in 1970, received his PhD from Mechanical Engineering Department, Ferdowsi University of Mashhad, Iran in 2010. His field of study is solidification in stagnation flow. During his $\mathrm{PhD}$ program, he established a three-dimensional computer program to predict the flow, temperature, and solidification of a fluid in stagnation flow. In 2011, he joined Payam Noor University of Mashhad as an Assis- tant Professor, teaching primarily CFD, advanced heat transfer, advanced numerical calculations, and engineering mathematics. He works on techniques in computer programs of modeling process in heat and fluid flow with phase change. His works include analytical and experimental methods. His main interests are solidification, phase change to liquid, heat, and fluid flow. He has already published about 10 international journal papers (ISI) and a book "Convective heat transfer".

Shima Yazdani was born in Shiraz, Iran in 1992. She received a BSc degree in Mechanical Engineering from Quchan University of Technology, Iran in 2014 and her MSc degree in Energy Conversion from Payame Noor University of Mashhad, Iran in 2017. She is currently a PhD student at Hakim Sabzevari University, Iran. Her main research interests include energy, computational fluid dynamics, and aerodynamics. She has already published two papers in international conferences and three journal papers. 